NASA/CR-1998-208735

ICASE Report No. 98-47

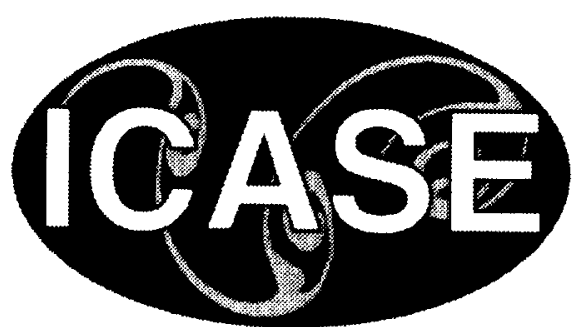

\title{
A Rigorous Framework for Optimization of Expensive Functions by Surrogates
}

Andrew J. Booker

Boeing Shared Services Group, Seattle, Washington

J.E. Dennis, Jr.

Rice University, Houston, Texas

Paul D. Frank

Boeing Shared Services Group, Seattle, Washington

David B. Serafini

E.O. Lawrence Berkeley National Laboratory, Berkeley, California

Virginia Torczon and Michael W. Trosset

The College of William and Mary, Williamsburg, Virginia

Institute for Computer Applications in Science and Engineering

NASA Langley Research Center, Hampton, VA

Operated by Universities Space Research Association

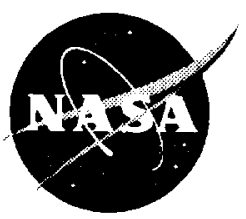

National Aeronautics and

Space Administration

Langley Research Center

Hampton, Virginia 23681-2199
Prepared for Langley Research Center under

Contracts NAS1-19480 and NAS 1-97046

November 1998 
Available from the following:

NASA Center for AeroSpace Information (CASI)

7121 Standard Drive

Hanover, MD 21076-1320

(301) 621-0390
National Technical Information Service (NTIS)

5285 Port Royal Rozd

Springfield, VA 22151-2171

(703) $487-4650$ 


\title{
A RIGOROUS FRAMEWORK FOR OPTIMIZATION OF EXPENSIVE FUNCTIONS BY SURROGATES
}

\author{
ANDREW J. BOOKER*, J. E. DENNIS, JR. ${ }^{\dagger}$, PAUL D. FRANK ${ }^{\dagger}$, DAVID B. SERAFINI ${ }^{\S}$, \\ VIRGINIA TORCZON $\uparrow$, AND MICHAEL W. TROSSET
}

\begin{abstract}
The goal of the research reported here is to develop rigorous optimization algorithms to apply to some engineering design problems for which direct application of traditional optimization approaches is not practical. This paper presents and analyzes a framework for generating a sequence of approximations to the objective function and managing the use of these approximations as surrogates for optimization. The result is to obtain convergence to a minimizer of an expensive objective function subject to simple constraints. The approach is widely applicable because it does not require, or even explicitly approximate, derivatives of the objective. Numerical results are presented for a 31-variable helicopter rotor blade design example and for a standard optimization test example.
\end{abstract}

Key words. approximation concepts, surrogate optimization, response surfaces, pattern search methods, derivative-free optimization, design and analysis of computer experiments (DACE), computational engineering.

Subject classification. Applied \& Numerical Mathematics

1. Introduction. The use of computer simulations in engineering decision-making is growing in importance. A prototypical example, described in Section 3, involves designing a low-vibration helicopter rotor blade. This example poses an optimization problem in which evaluation of the objective function requires running expensive analysis code(s). Existing methods for such optimization problems are either impractical or ad hoc. In this paper, we present a rigorous framework for optimizing expensive computer simulations through the use of inexpensive approximations of expensive analysis codes.

We will set forth, for comment and criticism, a rigorous approach to solving the following mathematical

*Mathematics \& Engineering Analysis, Bocing Shared Services Group, Applied Research and Technology, Box 3707, M/S 7L-22, Seattle, Washington 98124 .

† Department of Computational and Applied Mathematics \& Center for Research on Parallel Computation, Rice University, P. O. Box 1892, Houston, Texas 77005. This research was supported by the Department of Energy under grant FG0393ER25178, by the Air Force Office of Scientific Research under grant F49620-95 1-210, by the Center for Research on Parallel Computation under the National Science Foundation grant CCR 9120008, by the Boeing Company, and by the REDI Foundation.

${ }^{\ddagger}$ Mathematics \& Engineering Analysis, Boeing Shared Services Group, Applied Research and Technology, Box 3707, M/S 7L-21, Scattle, Washington 98124 .

$\S$ National Energy Research Scientific Computing Center, E.O. Lawrence Berkeley National Laboratory, MS 50B 2239, 1 Cyclotron Road, Berkeley, California 94720, <dbsenersc.gov>. This research was supported by the Air Force Office of Scientific Research under grant F49620-95 1 0210.

Department of Computer Science, College of William \& Mary, P. O. Box 8795, Williamsburg, Virginia 23187. This research was supported by the Air Force Office of Scientific Research under grant F49620 95-1 0210, by the National Science Foundation under grant CCR-9734044, and by NASA Contract Nos. NAS1-19480 and NAS1-97046 while the author was in residence at the Institute for Computer Applications in Sciencc and Engineering (ICASE), NASA Langley Research Center, Hampton, Virginia.

"l Department of Mathematics, College of William \& Mary, P. O. Box 8795, Williamsburg, Virginia 23187. This research was supported by the Air Force Office of Scientific Research under grant F49620 9510210 and by NASA Contract No. NAS1 19480 while the author was in residence at the Institute for Computer Applications in Science and Engineering (ICASE), NASA Langley Research Center, Hampton, Virginia. 
problem:

$$
\begin{aligned}
& \operatorname{minimize} f(x) \\
& \text { subject to } x \in \mathcal{B} \equiv\{x \mid a \leq x \leq b\},
\end{aligned}
$$

where $f: \Re^{n} \rightarrow \Re \cup\{\infty\}, a, b \in \Re^{n}$, and $a \leq b$ means that each coordinate satisfies $a_{i} \leq b_{i}$. The following characteristics distinguish the subset of such problems for which our methods are intended:

1. The computation of $f(x)$ is very expensive and the valies obtained may have few correct digits.

2. Even if $x$ is feasible, the routines that evaluate $f(x)$ mey fail to return a value at the same computational cost as if a value were returned.

3. It is impractical to accurately approximate derivatives of $f$.

4. If $x$ is infeasible, then $f(x)$ may not be available.

Typically, $f(x)$ is expensive to evaluate because there are large numbers of ancillary or system variables that must be determined for each choice of $x$ before $f(x)$ can te evaluated. For example, in the helicopter rotor blade design problem, each $x$ specifies a coupled system (f partial differential equations (PDEs) that must be solved in order to obtain dependent system variables required to evaluate $f(x)$. It may be quite difficult to obtain accurate solutions of such systems, even after expending substantial computational resources. Furthermore, when a coupled system of PDEs is solved by an iterative method, e.g., the notoriously unrcliable method of successive substitution, the method may fail to converge at all. Thus, one cannot assume even that one will obtain an objective function value at each feasible point.

The difficulties implied by the first two properties are conpounded if the intended optimization algorithm requires derivative information. Actual derivatives are rarely available, although we hope that this circumstance will change as automatic differentiation technology advances. On the other hand, choosing an appropriate step size for approximating derivatives by finite differences is itsclf a difficult undertaking. Moreover, the difficulties are compounded by the expense of function evaluation and the fact that a function value may not be returned.

The foregoing considerations lead us to dismiss the possibility of using traditional quasi-Newton methods to solve Problem (1). Indeed, even if actual derivative information were available, quasi-Newton methods might be poor choices because they are adversely affected by fu zction inaccuracies [15]. Instead, we observe that direct search methods $[14,38,41,40]$ do not require deriv atives and are relatively insensitive to function inaccuracies. Their shortcoming, especially when functior evaluation is expensive, is that in practice they tend to require a great many function values. The essential observation of the present paper is that inexpensive surrogate objective functions can be used to accelerate (certain methods of) direct search for a solution without sacrificing theoretical guarantees of asymptoti: convergence.

The use of direct search methods provides a natural way to $\varepsilon$ ddress the fact that $f(x)$ may be unavailable for some feasible $x$. When this occurs, we simply assign $f(x)=\infty$. This assignment implicitly assumes that $x$ is suboptimal if $f(x)$ is not available. In fact, the failure of $f(x)$ to evaluate might result from failures in the analysis code rather than from the physical suboptimality of the design $x$, but we do not attempt to distinguish these possibilities in the present paper.

If the optimization method causes us to consider an infersible $x$, then we decline to try to evaluate $f(x)$. In fact, it is common practice for optimization algorithms not to evaluate the objective at points that violate simple bound constraints because such violations are easily detected. In contrast, it is also common practice to evaluate the objective whenever the bound constr:ints are satisfied, regardless of the possible violation of more complicated (e.g., nonlinear equality) constraints. In the present paper, we skirt this issue 
by considering formally only bound constraints. Of course, we are kecnly aware that most problems also include other types of constraints, but the rigorous management of such constraints is a topic for future research - to consider it now would only cloud the issues that we wish to address in this forum. Thus, we treat the linear inequality constraints in our helicopter rotor blade test example by declining to evaluate $f(x)$ when $x$ is infeasible.

Problems of the type that we have described arise in disparate ways in engineering design and in manufacturing process control. Furthermore, there is a standard engineering practice [1] for attacking such problems:

1. Choose a surrogate $s$ for $f$ that is either

(a) a simplified physical model of $f$; or

(b) an approximation of $f$ obtained by evaluating $f$ at selected design sites, $x_{1}, \ldots, x_{d} \in \mathcal{B}$, at which each $f\left(x_{i}\right)$ is finite, then interpolating or smoothing the function values thus obtained.

2. Minimize the surrogate $s$ on $\mathcal{B}$ to obtain $x_{s}$.

3. Compute $f\left(x_{s}\right)$ to determine if improvement has been made over the best $x$ found to date, which may be some baseline $x$ or one of the design sites (if that approach is used).

The standard practice violates a fundamental tenct of numerical optimization, that one should not work too hard until one nears a solution. In fact, the standard practice is a one-shot approach: except for the final validation of $x_{s}$, all of the function evaluations are performed at sites selected by experimental design criteria with no concern for optimization per se. Furthermorc, this approach begs a potentially embarrassing question, viz., what does one do if (as is often the case) $x_{s}$ is not good enough to use as a solution to Problem $(1) ?$

A natural modification of the standard practice is to use a sequence of surrogates to identify promising regions in which to use successively better surrogates, cither by adopting models with greater physical fidelity or by constructing approximations from a greater concentration of design sites. Examples of this basic strategy include $[6,20,13]$.

We present here a general methodology inspired by ideas in [13]. Our methodology is built on top of a general class of direct search methods for numerical optimization, the pattern search methods. We exploit in a novel way the convergence analysis for pattern search methods presented in $[39,24,25]$. Key to our approach is the observation that the convergence analysis allows great flexibility in the heuristics that one can employ to find the next iterate. Accordingly, we perform a fairly extensive search on the current surrogate to select new points at which to evaluate the objective. In this paper, we are concerned with surrogates that are interpolating approximations of the objective and we use any new values that we obtain to update the current approximation.

In the next section, we present our surrogate management framework (SMF) and demonstrate that it works on a standard test problem from the global optimization literature [16]. In subsequent sections, we claborate on the earlier presentation by examining a problem for which the computational cost of evaluating the objective can be substantial. In Section 3, we describe the helicopter rotor blade design problem. In Section 4, we describe a family of interpolating approximations that has become popular in the literature on the design and analysis of computer experiments (DACE). In Section 5, we sketch some ways of using DACE approximations as optimization surrogates. Finally, in Section 6, we report some numerical results.

Some indications of how this work fits into a larger effort are provided in Sections 2.3 and 7.

2. A Rigorous Framework for Optimization Using Surrogates. In this section we describe SMF, our framework for managing surrogate objective functions to facilitate the optimization of expensive com- 
puter simulations. The framework is sufficiently general to accc mmodate surrogates that are (1) simplified physical models of the expensive simulation; (2) approximations of the expensive simulation, constructed by interpolating or smoothing known values of the objective; or (3) model-approximation hybrids. For the sake of clarity, however, we focus on the case of surrogates of the si cond type. In Section 4 we will emphasize intcrpolating approximations constructed by kriging, but the simple example in Section 2.3 illustrates that SMF also works with polynomial interpolants. Another example: using polynomial interpolants is presented in $[32]$.

We begin, in Section 2.1, by describing the family of underlying optimization algorithms on which SMF is based. Next, in Section 2.2, we formally define SMF. We onclude, in Section 2.3, by using SMF to minimize a simple algebraic test function.

2.1. Pattern Search Algorithms. Pattern search algorithms are a class of direct search methods for numcrical optimization. A formal definition of pattern search, wilich includes various well-known algorithms, was proposed in [39]. An elementary introduction to pattern search algorithms and a discussion of their historical antecedents is available in [40].

Pattern search algorithms are characterized by two crucial notions, a sequence of meshes and a list of polling conditions. A mesh is a lattice to which the search $\mathrm{fcr}$ an iterate is restricted. As optimization progresses, the polling conditions govern when the current mesh can be refined, ensuring that the algorithm will satisfy the demands of the convergence theory for pattern search methods.

For our purposes, the primary polling condition that must be enforced to ensure convergence is that the set of vectors formed by taking the differences between the set of trial points at which the objective function is to be evaluated (the pattern) and the current iterate $x_{k}$ must contain a positive basis for $\Re^{n}$. A positive basis [11] is a set of vectors whose nonnegative linear combinations span $\Re^{n}$, but for which no proper subset has that property. For our purposes, the relevance of a positive basis is that it ensures that if the gradient of $f$ at $x_{k}$ is not zero, then at least one vector in the positive ba is defines a descent direction for $f$ from $x_{k}$. This can be guaranteed without any knowledge of the gradient. Any positive basis has at least $n+1$ and at most $2 n$ vectors; we call these minimal and maximal positive bises, respectively.

For unconstrained problems, a minimal positive basis is sufficient to guarantee convergence [25]. However, for problems with rectangular feasible regions, c.g., Problem (1), we use a maximal positive basis that comprises all of the coordinate directions, both positive and negative. This guarantees that it is possible to move along the boundary of the feasible region and thus prevents premature convergence to a point that is not a constrained stationary point [24]. Recent work [26] tas revealed that it is possible to construct adaptive pattern search algorithms that identify only those con straints that are either binding or "almost" binding at the current iterate so that the number of vectors nee ded at any given iteration can vary between $n+1$ and $2 n$, inclusively.

The following formulation of Generalized Pattern Search (CPS) differs from the formulation of pattern search in $[39,24,25,26]$, but it is especially well-suited to our presentation. We remind the reader that if $f(x)$ either is infeasible or cannot be evaluated successfully, the 1 we set $f(x)=\infty$.

GPS: Let $M_{0}$ denote a mesh on $\mathcal{B} \equiv\{x \mid a \leq x \leq b\}$ and suppc se that $x_{0} \in M_{0}$ has been given. (In typical practice, $x_{0} \approx x^{*}$, where $x^{*}$ is a preliminary baseline solution, sut any choice of $x_{0} \in M_{0}$ is possible.) Let $X_{0} \subset M_{0}$ contain $x_{0}$ and any $2 n$ points adjacent to $x_{0}$ for which the differences between those points and $x_{0}$ form a maximal positive basis (composed of multiples of the ccordinate vectors) for $\Re^{n}$. As the algorithm generates $x_{k} \in M_{k}$, let $X_{k} \subset M_{k}$ be defined in the same way. For $k=0,1, \ldots$, do: 
1. Search: Employ some finite strategy to try to choose $x_{k+1} \in M_{k}$ such that $f\left(x_{k+1}\right)<f\left(x_{k}\right)$. If such an $x_{k+1}$ is found, declare the Search successful, set $M_{k+1}=M_{k}$, and increment $k$;

2. else Poll:

if $x_{k}$ minimizes $f(x)$ for $x \in X_{k}$, then declare the Poll unsuccessful, set $x_{k+1}=x_{k}$, and refine $M_{k}$ to obtain $M_{k+1}$ by halving the mesh size (write this as $M_{k+1}=M_{k} / 2$ );

else declare the Poll successful, set $x_{k+1}$ to a point in $X_{k}$ at which $f\left(x_{k+1}\right)<f\left(x_{k}\right)$, and set $M_{k+1}=M_{k}$.

Increment $k$.

Step 2 provides the safeguard that guarantees convergence, as in the following result [24].

Theorem 2.1.1. If $f$ is continuously differentiable on the feasible region $\mathcal{B}$, then some limit point of the sequence $\left\{x_{k}\right\}$ produced by a generalized pattern search (GPS) method for bound constrained minimization is a constrained stationary point for problem (1).

Notice that this result guarantees that GPS will converge no matter how naive the search strategy in Step 1. In practice, of course, the sophistication of the search strategy matters a great deal. We now turn to SMF, which uses surrogate objective functions to try to Search with greater parsimony and thereby reduce the total number of objective function evaluations.

2.2. The Surrogate Management Framework. The description of SMF that we present here is a set of strategies for using approximations in both the Search and Poll steps of a GPS algorithm. For greater clarity, we have also identificd a separate Evaluate/Calibrate step. In what follows, we assume that a family of approximating functions has been specified, that an initial approximation has becn constructed, and that an algorithm to recalibrate the approximation is available.

SMF: Given $s_{0}$, an initial approximation of $f$ on $\mathcal{B}$, and $x_{0} \in M_{0}$, let $X_{0} \subset M_{0}$ contain $x_{0}$ and any $2 n$ points adjacent to $x_{0}$ for which the differences between those points and $x_{0}$ form a maximal positive basis (composed of multiples of the coordinate vectors) for $\Re^{n}$. As the algorithm generates $x_{k} \in M_{k}$, let $X_{k} \subset M_{k}$ be defined in the same way. For $k=0,1, \ldots$, do:

1. Search: Use any method to choose a trial set $T_{k} \subset M_{k}$. If $T_{k} \neq \emptyset$ is chosen, then it is required to contain at least one point at which $f(x)$ is not known. If $T_{k}=\emptyset$, then go to Poll.

2. Evaluate/Calibrate: Evaluate $f$ on elements in $T_{k}$ until either it is found that $x_{k}$ minimizes $f$ on $T_{k}$ or until $x_{k+1} \in T_{k}$ is identified for which $f\left(x_{k+1}\right)<f\left(x_{k}\right)$. If such an $x_{k+1}$ is found, then declare the Search successful. Recalibrate $s_{k}$ with the new values of $f$ computed at points in $T_{k}$.

3. If Search was successful, then set $s_{k+1}=s_{k}, M_{k+1}=M_{k}$, and increment $k$; else return to Search with the recalibrated $s_{k}$, but without incrementing $k$.

4. Poll:

If $x_{k}$ minimizes $f(x)$ for $x \in X_{k}$, then declare the Poll unsuccessful, set $x_{k+1}=x_{k}$, and set $M_{k+1}=M_{k} / 2$;

else declare the Poll successful, set $x_{k+1}$ to a point in $X_{k}$ at which $f\left(x_{k+1}\right)<f\left(x_{k}\right)$, and set $M_{k+1}=M_{k}$.

Recalibrate $s_{k}$ with the new values of $f$ computed at points in $X_{k}$. Set $s_{k+1}=s_{k}$.

Increment $k$.

We structure our discussion of SMF around the proof of the following corollary of Theorem 2.1.1. Notice that this result assumes nothing about the accuracy of the approximations. In practice, of course, we would 
expect better approximations to yield better results.

Theorem 2.2.1. If $f$ is continuously differentiable on the fea ible region $\mathcal{B}$, then some limit point of the sequence $\left\{x_{k}\right\}$ produced by SMF for bound-constrained minim zation is a constrained stationary point for problem (1).

Proof: The proof is accomplished by showing that SMF is an instance of a generalized pattern search method and so Theorem 2.1.1 applies.

First, we need to be sure that we have specified a finite Search step, i.e., that there is a fixed upper bound on the number of unsuccessful search steps that will be tried before a poll step is taken. This follows immediately because each choice of $T_{k} \neq \emptyset$ must contain at lesst one point of $M_{k}$ at which $f$ is unknown and $M_{k}$ is a mesh on a compact set $\mathcal{B}$, hence a finite set.

We finish the proof by noting that the Poll step is still intact. From the perspective of the optimization algorithm, Poll is functionally unchanged by the recalibration step that it now includes.

The key to a successful implementation of SMF is to define the Search strategy in a way that efficiently exploits the current approximation $s_{k}$. One obvious approach is to search for points that minimize $s_{k}$. In [42], for cxample, a finite-difference quasi-Newton method was started from the current iterate with $s_{k}$ as the objective function. A more ambitious strategy would be to explore $s_{k}$ globally for multiple prospective basins, e.g., by performing a comprehensive grid search. For the examples in this paper, we performed a comprehensive search on a subset of the current mesh, $M_{k}$.

Notice that we do not require $f$ to be evaluated at all poirts in $T_{k}$ or $X_{k}$ before declaring a successful Search or Poll step. Once we have identified a point in either $T_{k}$ or $X_{k}$ with an objective value strictly less than $f\left(x_{k}\right)$, we can declare the iteration to be successful and ircrement $k$. This practical flexibility derives from a powerful and crucial aspect of the convergence theory developed in $[39,24,25,26]$ : it is not necessary for a pattern search algorithm to find the best point on the current mesh $M_{k}$, or even the best point in $X_{k}$ any point that produces decrease on $f\left(x_{k}\right)$ will suffice.

The convergence theory states that any point on the current mesh, $M_{k}$, that produces decrease on $f\left(x_{k}\right)$ can be used as the next iterate, $x_{k+1}$. Traditional pattern search algorithms evaluate $f$ at a predetermined subset of $M_{k}$ (a pattern) in order to try to discover such points. To try to reduce the number of function evaluations required to discover a point that produces decrease, SMF uses the current approximation to predict points in $M_{k}$ at which we expect to realize decrease. The set $T_{k}$ contains our list of potential candidates. If $T_{k}$ contains multiple candidates, then we choose those that are considered most promising. If the approximation does not predict any such decrease, then we may choose to set $T_{k}=\emptyset$ and Poll.

SMF affords complete flexibility in deciding how many points to include in $T_{k}$. One obvious possibility is to include a single point: the one at which the surrogate predicts the greatest decrease on $f\left(x_{k}\right)$. This is preciscly what we did to obtain the results reported in Section 2.3. In other situations, however, it may be desirable to include several points. One such circumstance aris es when attempts to evaluate the objective are prone to failure. For example, for the helicopter rotor bla de design problem we have performed runs in which $60 \%$ of our attempts to evaluate $f$ at a feasible $x$ faied. Thus, to obtain the results reported in Section 6, we choose $T_{k}$ to contain three points in an effort to ensure that at least one of the points in $T_{k}$ can be evaluated successfully. Another circumstance arises in parallel or distributed computing environments. If several processors are available to perform simultaneous function evaluations, then it is natural to provide $T_{k}$ with one point for each available processor. 
We also allow $T_{k}$ to contain points at which the approximation does not predict decrease. This flexibility is desirable because the step Evaluate/Calibrate actually serves two purposes. On the one hand, we obviously want to find an $x_{k+1} \in T_{k}$ for which $f\left(x_{k+1}\right)<f\left(x_{k}\right)$. On the other hand, after each step we know more objective function values and we therefore compute a new (and presumably more accurate) approximation. However, the points that most decrease the objective may not be the points that most improve the accuracy of the approximation. In fact, it may be desirable to select trial points that balance the competing goals of decreasing the objective and constructing a better approximation. By selecting trial points that lead to better approximations, we may gain greater insight into the global behavior of $f$, accelerating optimization by improving the quality of future searches. This idea will be discussed in Section 5.

Except for recalibration, the Poll steps for GPS and SMF are identical. Although SMF does not specify the order in which $f$ is to be evaluated at the points in $X_{k}$, it is natural to evaluate them in increasing order of the objective values predicted by $s_{k}$.

If the current iterate is sufficiently near a minimizer of the true objective function, then the current mesh must be refined in order for optimization to progress. It is the Poll step that guarantees convergence, but this guarantee can be costly. For bound-constrained optimization, an unsuccessful Poll step requires between $n$ and $2 n$ evaluations of the objective function [25], so we would like to avoid Poll steps whenever possible. One possibility is to employ a hybrid approach that starts with SMF and assumes that an unsuccessful Poll step signals a basin of attraction for a local method, which we then call to see if it can succed. Of course, we might need to revert to SMF if we switch too hastily. A natural candidate for the second phase of such a hybrid approach is the derivative-free optimization (DFO) algorithm described in $[8,7]$. The development of an SMF-DFO hybrid is one objective of the larger effort mentioned in Sections 2.3 and 7.

2.3. Sample Test Results. We now apply SMF to a standard global optimization test problem, the six-variable Hartman problem [16], which has a single global minimizer and several nonglobal minimizers. In realistic applications, objective functions are expensive to cvaluate and computed values have only several digits of accuracy. Hence, we only attempt to solve the Hartman problem approximately, and we are prepared to decrease the chance of converging to the global minimizer in order to restrict the total number of objective function evaluations.

Figure 1 presents run histories for two implementations of SMF, as well as the final values obtained from nine runs of the DFO algorithm described in $[8,7]$. We imposed bounds of $\mathcal{B}=[0,1]^{6}$ and started each run from $x_{0}=(.5, .5, .5, .5, .5, .5)^{T}$. (The nine runs of DFO produced different results because the DFO algorithm includes a stochastic decision.) The only difference between the two implementations of SMF is the choice of approximating families: one choice interpolated known function values with variable-order multivariate polynomials [12] whose degrees were increased as more function values were obtained; the other choice interpolated known function values by kriging. The latter family of approximations, which we also used for the helicopter rotor blade design problem, is discussed in Scction 4. In each implementation, the initial approximation was constructed by interpolating the same set of 16 known function values. Each time that Search was called, the current approximation was evaluated on a 7280-point subset of the current mesh. Because the Hartman objective can always be evaluated, Search returned only a single point from the subgrid at which the approximation predicted the grcatest decrease in the Hartman objective.

The global minimum of the Hartman function is -3.322. Except for one run of DFO, each run produced (approximately) this value. Of particular note is the fact that SMF worked effectively with each of two different families of approximations. The SMF run histories exhibit the characteristic plateaus that result 
when Poll stcps are executed on a sequential computer. The actual ("wall clock") time spent on these steps can be reduced if opportunities for parallel or distributed computing exist. If a sufficient number of processors are available to evaluate all of the required function values concurrently, and if the abscissa indicates actual time rather than number of function evaluations, then the plateaus usually disappear.

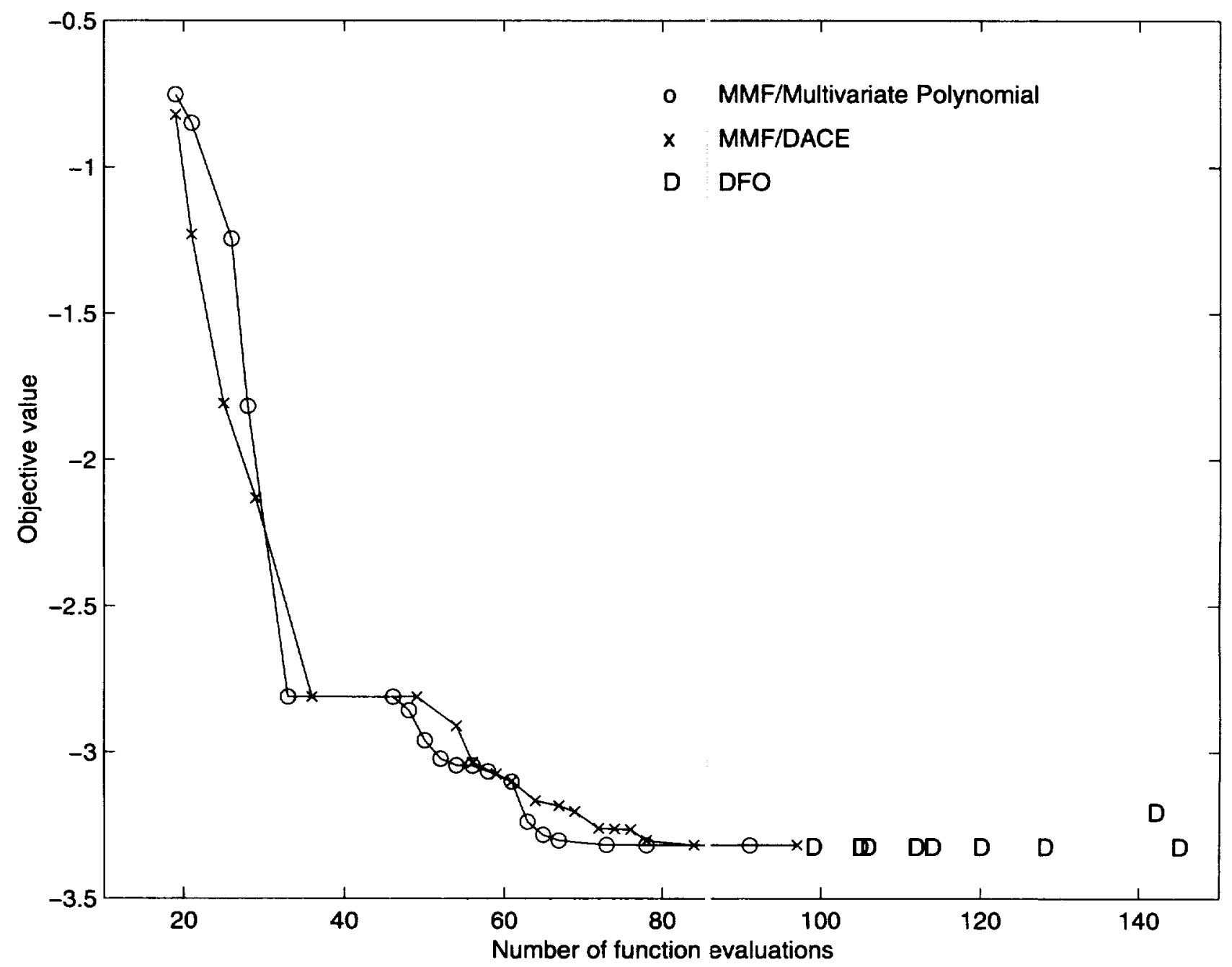

FIG. 1. Results for the six variable Hartman problem

3. The Helicopter Rotor Blade Design Problem. Helicopter rotor blade design is used here for illustrating concepts and motivating algorithmic requirements fur SMF applied to industrial problems. The particular task we consider is structural design of helicopter rotc $r$ blades for minimum vibration transmitted to the hub.

As indicated in Figure 2, the design variables consist of up to five structural parameters for each span segment. The variations on this problem that we have considerec have between 10 variables and 56 variables. As described below the objective function is a weighted sum o: various harmonics of forces and moments. The analysis code used is Tech01 [33].

Tech01 is a multidisciplinary analysis code. The disciplines include dynamic structures, acrodynamics, 


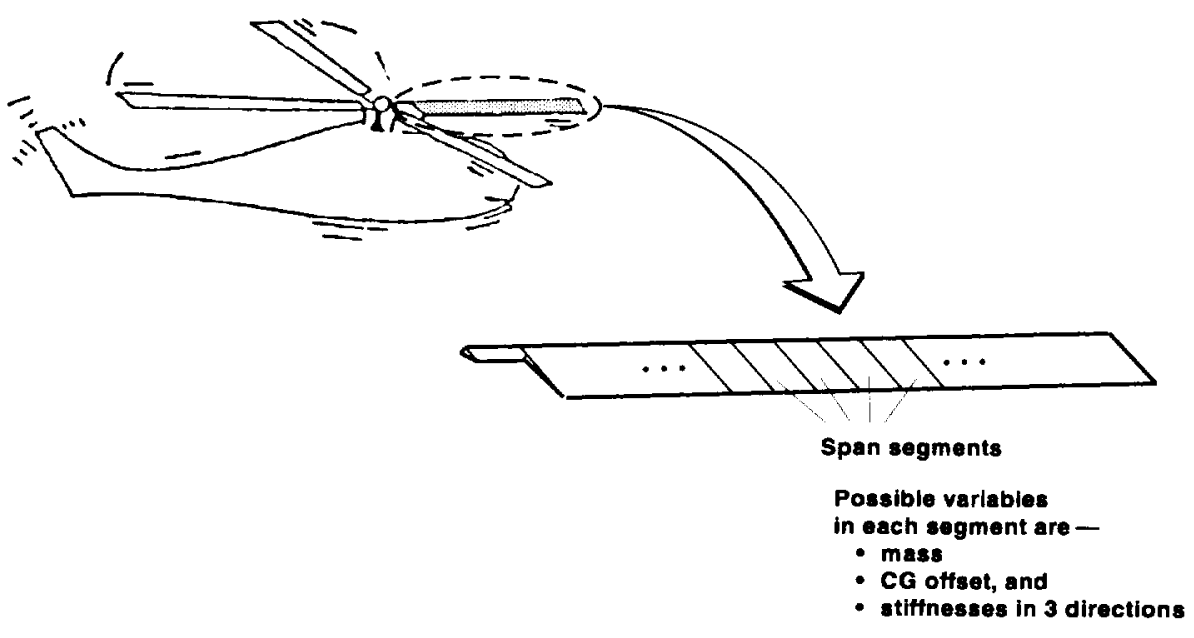

FIG. 2. Rotor blade design variables

wake modeling, and controls. The run time for a Tech01 fixed-wake analysis is roughly 20 minutes on a midlevel workstation. However, the run time can increase to several days on the same machine if wake updating is invoked. The full wake analysis has greater fidelity to the physics of the problem. Our main focus is on the use of approximations to the analysis code results as objective function surrogates for optimization. Thus, to facilitate studies of algorithmic issues for surrogate optimization, the test results discussed here use fixed-wake analyses.

A more detailed statement of the optimization problem is

$$
\begin{array}{ll}
\text { minimize } & f(x)=\sum_{i=1}^{n h} w_{i} \frac{\left|h_{i}(x)\right|}{\left|h_{i}\left(x_{B}\right)+1\right|} \\
\text { with respect to } & x \in \Re^{n} \\
\text { subject to } & \mathrm{xu}_{j} \geq x_{j} \geq \mathrm{xl}_{j}, j=1, \ldots, n \\
& \mathrm{cu}_{k} \geq c_{k}(x) \geq \mathrm{cl}_{k}, k=1, \ldots, \text { ncon. }
\end{array}
$$

In the above equation, the $h_{i}, i=1, \ldots, n h$, are output responses from Tech01. The subscript $i$ is an index that maps from the response function vector elements to forces in three directions, moments in three directions, and harmonic numbers for each force and moment. In addition, the indexing may span several flight conditions, such as hover and forward flight at various speeds. Normalization to account for the different physical units of the responses is accomplished by including $h_{i}\left(x_{B}\right)$ in the denominator of the objective function terms, where $x_{B}$ is the baseline design.

The objective function components are weighted by factors $w_{i}$. The limits $\mathrm{xu}_{j}, \mathrm{xl}_{j}, \mathrm{cu}_{k}$, and $\mathrm{cl}_{k}$ are upper and lower bounds on the variables and constraints, respectively. The constraints $c_{k}(x)$ can be quantities such as required rotor horsepower, centrifugal force, autorotational inertia, snow load, and limits on total mass. Aside from the bounds on the independent variables, the only constraint in the examples considered here is total mass. Since the masses are a subset of the design variables, the mass constraint is a linear constraint involving a subset of the variables. Thus, it is independent of the analysis results, and does not require consideration of issues involving the construction of surrogate approximations of constraint functions.

The helicopter rotor blade design problem is summarized in Table 1. Note that this problem has upper and lower bounds on all the variables. 


\begin{tabular}{|c|c|c|c|}
\hline Example & Variables & Objective & Constraints \\
\hline 31 Variable & $\begin{array}{l}10 \text { masses, } \\
10 \text { centers of gravity, } \\
11 \text { stiffnesses in } \\
\text { a single direction }\end{array}$ & $\begin{array}{l}\text { weighted sum of } 1 \mathrm{st} \\
\text { and } 2 \text { nd hurmonics } \\
\text { for two flicht } \\
\text { conditions }\end{array}$ & $\begin{array}{l}\text { upper bound on } \\
\text { sum of masses }\end{array}$ \\
\hline
\end{tabular}

4. Constructing the Surrogates. Even with fixed wake, the helicopter rotor blade design examples are not easily modeled using simplified, less expensive simulations. In consequence, we concentrate on approximations constructed by interpolating or smoothing a set of known objective function values. In this section we describe our method of choosing a set of initial design sites at which the objective function $f$ is cvaluated before optimization commences, our choice of a class of function approximations from which the initial surrogate $s$ is to be selected, our method of selecting $s$ from this class, and some diagnostic procedures for extracting useful information from $s$.

The problem of choosing a set of initial design sites, $x_{1}, \ldots, x_{d} \in \mathcal{B}$, is a problem in the design of experiments. This problem has been studied extensively in the recent litcrature on the design and analysis of computer experiments (DACE), surveys of which include $[31,2,22]$.

We seek designs that are "spacc-filling" (for lack of a better term), i.e., that will allow us to sample the behavior of the objective function throughout the feasible region. We want to avoid designs that are ticd to a narrow class of approximating functions, e.g., linear or quadratic functions. We want to be able to generate designs somewhat automatically, and we would like to be able to generate designs for irregular (nonrectangular) feasible regions.

We have opted for designs that are used in quasi-Monte Carlo integration: Latin hypercube sampling (LHS) $[27,35]$, orthogonal arrays $(\mathcal{O A})[28]$ and $\mathcal{O} \mathcal{A}$-based LHS [36]. In LHS, each of the $n$ variables is chosen from $d$ equally spaced values. The $\mathcal{O}$ ss we use are spac--filling in the following sense: the variables in the experimental design are assigned from $l$ distinct values. In every subset of $k$ variables every one of the $l^{k}$ combinations of values occurs the same number of times. Arrays with this property are of strength $k$. LHS designs are of strength 1 . Typically we use $\mathcal{O}$ As of strength 2 . This is a straightforward process because LHS designs are easily generated and efficient code for generating $\mathcal{O A}$ designs is available from STATLIB (http://lib.stat.cmu.edu).

LHS and $\mathcal{O A}$ were devised for rectangular regions. In the helicopter rotor blade design examples, the mass constraint induces a nonrectangular feasible region. We hitve experimented with various strategies for adapting $\mathcal{O} \mathcal{A}$ designs to this region, e.g.,

- Generate a design with $d$ points in the rectangle defined yy the variable bounds, then alter the design so that the $d$ points satisfy the mass constraint.

- Generate a design with many points in the rectangle defined by the variable bounds, then discard the points that are outside a slightly expanded mass constraint boundary.

After the design sites have been chosen and the objective unction $f$ has been evaluated at them, the initial surrogate $s$ can be constructed. This surrogate is intended to be an approximation of $f$ throughout the region of interest that is inexpensive to evaluatc. It will be reca ibrated as new function values are obtained in the course of solving the optimization problem. Because we do not want to make a priori assumptions about the structure of $f$, we require a large, flexible class of functions from which surrogates can be selected. 
Plausible families of approximating functions include neural networks and low degree interpolating polynomials [12]. In $\S 2.3$ we gave evidence that the SMF can use different families of approximation. We have opted for the family of functions defined by the kriging procedures discussed in the DACE litcrature. The kriging parameterization, defined by means and covariances of function values, is more intuitive for the present applications than other approximations in the response surface literature. For some choices of covariance function, kriging is equivalent to spline interpolation, a correspondence that has been discussed in the geostatistics literature [43].

It is quite common in the statistics literature to motivate kriging by assuming that $f$ is a realization of a stationary Gaussian spatial process. As implausible as this assumption may seem in the present context, it does suggest useful ways to proceed with the selection of a surrogate objective function from the family of approximating functions. Upon making this assumption, it becomes possible to estimate mean and covariance parameters from $f\left(x_{1}\right), \ldots, f\left(x_{d}\right)$ by the method of maximum likelihood estimation (MLE) and thereby to specify a well-defined procedure for selecting $s$.

Although MLE has been criticized in the spatial statistics literature, e.g. [30], it has been defended by others as a crude form of cross-validation $[19,10]$. Our experience to date has been similar to that reported in [31]: "crude MLE's lead to useful prediction...." Assuming that the covariances in question are a constant unknown variance times unknown correlations of a specified form, there exist closed-form expressions for the MLEs of the mean and variance parameters. To obtain MLEs of the correlation parameters, we have attempted global optimization of the (log) likelihood function via an implementation of the algorithm in [29].

One technical difficulty with kriging should be noted. Kriging calculations require inversion of the matrix of estimated correlations between function values at the design sites. The initial correlation matrix usually is well-conditioned but as the function is sampled at additional sites that cluster near a minimizer, the process of recalibration gencrally causes subsequent correlation matrices to become ill-conditioned. We have addressed this difficulty by adding a small number $\left(10^{-6}\right)$ to the diagonal of the correlation matrix. With this addition, the approximating functions do not exactly interpolate the observed function values; however, they retain their flexibility and predict observations very closely.

Once a surrogate function $s$ has been constructed, one can use it to predict values of $f(x)$ and also to approximately bound the errors in such predictions. The latter is accomplished by calculating mean squared crror (MSE) under the assumption of a stationary Gaussian process. It has been argued in [31, 21] that this is a reasonable framework in which to bound future prediction errors, particularly if one can assess the plausibility of the assumption of a stationary Gaussian process. Since larger values of MSE are associated with larger uncertainty in prediction, we have used MSE to guide our choice of new sites at which data would be of particular value in improving the accuracy of the surrogate.

One also might predict future prediction errors by examining the cross-validation residuals. These error estimates are obtained at each observation by kriging (with the original MLE parameters) the other observations and predicting the designated observation. Similarly, it was suggested in [21] that one might cross-validate the MSEs to assess their predictive capabilities.

Finally, we have found that performing a functional analysis of variance $[17,28,31]$ on the surrogate function $s$ is a useful way of identifying lower-dimensional subspaces in which most of the variation in $s$ resides. This ANOVA technique, which can in principle be applied to any square-integrable function, decomposes $s$ into main effects (contributions of individual variables to variation in $s$ ) and interaction effects (contributions of combinations of variables to variation in $s$ ). The hope is that one can identify a few 
key variables that account for most of the variation in $f$, then o timize solely with respect to those variables at reduced expense.

5. DACE Model Refinement by Balanced Searches. The Search phase of SMF allows us to use any method to choose a trial set of new mesh points at which to evaluate the true objective function. In this section we discuss several search strategies with which we have experimented.

Whatever approximations are used as surrogates for the ubjective function, a straightforward search strategy is to optimize (either locally or globally, depending on one's goals) the current approximation and to return trial points that reside on the mesh $M_{k}$ near the solusion(s) thus obtained.

A simple implementation of this strategy, in which a finit:-difference quasi-Newton method was used to find a local minimizer of the current DACE approximatior (see §4), is the model-assisted grid search (MAGS) described in [42]. MAGS was intended for situations in which only a relatively small number of function evaluations are permitted. Because it approximates the objective function over the entire feasible region, recalibration of the approximation is made using one new objective value at a time, as these values are produced by the optimization procedure.

In contrast to MAGS, a "zoom-in" method for local refinement was proposed in [18]. This strategy uses the existing approximation to determine an interesting subregion of the design space for further exploration. The optimization process is halted, additional function values are obtained in the subregion, and a new approximation is formed. The expectation is that the approxination constructed in the subregion will be more accurate than the original approximation because it will be based on a higher density of data.

One method for determining the zoom-in region is to locase the local minima of the original approximation and determine the extent of their basins. For a specified value greater than the function value at a local minimum, the extent of its basin is assessed in terms of the distance of the minimum in each coordinate direction to the nearest level set corresponding to the specified value (or the distance to the coordinate bound if the specified level is not attained.)

Zoom-in methods favor exploration in the vicinity of local sclutions of the current approximation. Hence, they may fail to find basins of better local solutions elsewhere in the design space. In the parlance of global optimization, they are purely local in nature. A purely global raethod for determining new points at which to cvaluate $f$ is to minimize an estimate of the integrated mean squared error (IMSE, [31]) of the resulting new approximation. This method is space-filling in that it terds to place new design points in previously unexplored regions. In contrast to zoom-in methods, the IMSE optimal methods defer examining promising regions in the interest of obtaining a better "global" picture of $t$ te design space. The result is that they tend to converge slowly to a minimizer of the objective.

To address both local and global concerns, we have experir nented with a balanced search strategy. This method is based on our observation that, at any location in design space, the current DACE approximation can supply two key pieces of information: an approximate va ue of the objective and an estimate of the approximation's mean squared error (MSE) at the point. Thə former provides purely local information; the latter, which increases with the distance from the subject f oint to the nearest design site and with the degree of nonlinearity of the data, quantifies uncertainty about the behavior of the true objective function and hence provides some degree of global information.

Based on local concerns, one would cvaluate $f$ at points that the approximation indicates have low values. Based on global concerns, one would evaluate $f$ at pints with high MSE values. The balanced search method selects a portion of the total of the trial set $T_{k}$ based on each measure. Ideally, one would measure the approximate values and MSEs at each point on a fine grid in design space and select the best 
candidates, as in [9]. Unfortunately, in high-dimensional design spaces it is impossible to consider even a crude grid formed by splitting each dimension in two; hence, the balanced search algorithm described below considers each member of a "dense cloud" of (say 5000) trial sites. To ensure that this cloud is space-filling, it is generated from an $\mathcal{O A}$-based LHS.

\section{Balanced Search Algorithm for New Site Selection}

Given: an existing approximation, current design sites, a list of local minimizers of the current approximation, the number nlocal of new design sites to be based on local concerns, the number nglobal of new design sites to be based on global concerns, and a tolerance $\tau$ equal to the minimum distance that will be allowed between any two sites.

1. Create an initial list of (say) 5000 trial sites using an $\mathcal{O A}$-based LHS.

2. Add the local minimizers of the current approximation to the list of trial sites.

3. Calculate the distances from each of the trial sites to each other and to each of the design sites for the current approximation.

4. Sort the trial sites in order of increasing values as detcrmined by the current approximation.

5. Select as new design sites the nlocal trial sites with the smallest values determined by the current approximation, maintaining the condition that each design site is $\geq \tau$ distant from every other design site.

6. Compute the MSE at each of the remaining trial sites using the correlation parameters of the current approximation, but after updating the model parameters to reflect MSE $=0$ at the newly selected design sites.

7. For $i=1, \ldots, n g l o b a l$, do:

(a) Select as a new design site the trial site with the largest MSE value, maintaining the condition that each design site is $\geq \tau$ distant from every other design site.

(b) Update the approximation to reflect $\mathrm{MSE}=0$ at the newest design site, then recompute the MSE at each of the remaining trial sites.

6. Test Results for Rotor Blade Design. We now summarize the performance of several optimization methods when applied to the helicopter rotor blade design problem described in Scction 3. We remind the reader that this problem has a linear inequality constraint which we treat by declining to evaluate $f(x)$ for any infeasible $x$. The optimization methods that we considered are the following:

- MMF: This is Serafini's [32] implementation of the surrogate management framework, SMF, described in Section 2.2. An initial approximation was constructed from 59 successful function evaluations using the DACEPAC software package $[2,3]$. The initial iterate was a baseline solution provided by Bocing. Search evaluated the current approximation on a 29,800-point subset of the current mesh and returned the three points with the lowest values. The true objective function was then evaluated sequentially at each of these points until one was found to be better than the current iterate.

- DFO: This is the derivative-free optimization method discussed in $[7,8]$. The results that we report, which include final values but not run histories, were provided by Katya Scheinberg. The initial iterate was the baseline solution provided by Boeing. Two variants of DFO were implemented, one that scales the decision variables to be of comparable magnitude and one that leaves the decision variables unscaled. (The significance of this distinction will be discussed below.) Because DFO randomly chooses the second point at which the objective function is evaluated, multiple runs of 
each variant were performed (nine for the scaled varian:, ten for the unscaled variant).

- PDS: This is Torczon's implementation [37] of the paralel direct search method of [14], with modifications by Serafini to support constraints and the standard Message Passing Interface (MPI) parallel communications library [34]. The initial iterate was the baseline solution provided by Boeing. PDS was executed using 96 evaluations of the objective pe: iteration, more than the minimal number (62) required to ensure convergence.

- GA: This is a genetic algorithm from PGAPack [23]. On the advice of its author, David Levine of the Bocing Company, we used a steady-state reproductive strategy with a population size of 200 and a replacement rate of $10 \%$ of the population per iteration.

- BLGS: This implementation of the SMF is due to Booker and Frank [4] and was discussed in Section 5. The initial approximation was the same as for MMF. The current approximation was refined twice, each time by adding 50 new values of the objective function. Some of these 50 new sites were chosen because the current approximation predicted that they would have objective function values lower than that of the current iterate; others were chosen because they were relatively far from any previously selected sites.

- Sampling: This is a simple sampling algorithm that generates $\mathcal{O} \mathcal{A}$-based LHS of the Bose type [28]. Each sample contains the initial design, and in addition, samples that contain 58, 200,380, and 684 convergent points were gencrated independently. For each sample, the best value of the objective function was taken to be the minimum of the objective function values computed at the points in that sample, if this value improved on the best found in prior samples. We include these results only as a simple strawman, and for this reason we did not count the rather larger number of points in each sample for which the $f(x)$ did not return a value, nor did we try to implement a more sophisticated sampling algorithm.

For each of the above optimization methods, the best objective function value obtained after selected numbers of function evaluations was plotted against the number of function evaluations. The resulting graph, adapted from [5, 32], is displayed in Figure 3. We report the total number of attempts to evaluate the objective function, whether or not the attempt was successful. However, we did not count unsuccessful attempts encountered during the construction of initial approximations, srior to commencement of the optimization algorithm. Thus, for MMF and BLGS, our count includes the 59 successful function evaluations obtained by DACEPAC, but not the additional 97 evaluation attempts t at failed. For the sampling algorithm, only successful function evaluations were counted.

The results summarized in Figure 3 are quite encouraging so good, in fact, that it may be that the 31-variable helicopter rotor blade design problem is substantial y easier to solve than we anticipated. Both GA and PDS performed as advertised. GA produced substanti 1 decrease with a small number of function evaluations, but then had difficulty descending below a fairly high value of the objective function. PDS descended somewhat more steadily to an appreciably lower value of the objective function. Both DFO and MMF found even lower objective function values in a nuuber of function evaluations that would be considered extremely small for finite-difference quasi-Newton m sthods.

Except for one variant of DFO, all of the algorithms for whic $h$ we have reported results scale the decision variables to be of comparable magnitudes. The variables in tile 31-variable helicopter rotor blade design problem differ by ten orders of magnitude, yet the single lowest value of the objective function was found by the variant of DFO that did not scale the variables. This appasent paradox deserves further comment. 


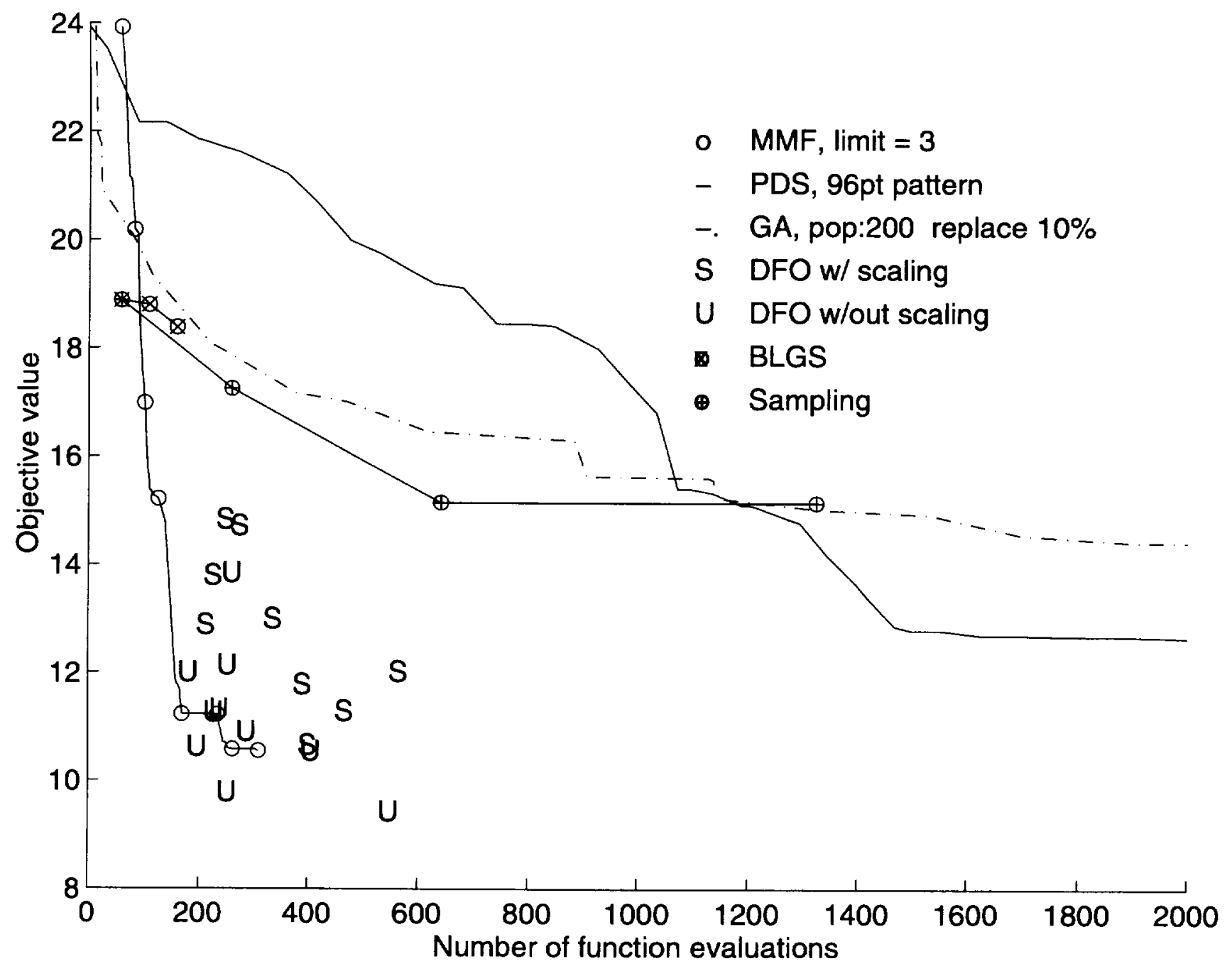

FIG. 3. Results for the 31-variable helicopter rotor blade design problem

When DFO is applied to the unscaled problem, its trust region precludes appreciable change in the variables of large magnitude but hardly restricts changes in the variables of small magnitude. In effect, DFO thereby restricts its search to the subspace defined by the variables of small magnitude. A subsequent ANOVA decomposition of a DACE approximation of the 31-variable helicopter rotor blade design objective function revealed that the objective does not vary much with respect to the variables of large magnitude. Thus, the unscaled variant of the DFO implementation was actually solving a lower-dimensional problem coincidentally generated by the most important variables. (This is a dramatic illustration of the diagnostic value of the ANOVA decomposition.) We are now investigating the lower-dimensional problem in greater detail.

Finally, we observe that most of the above algorithms can exploit parallelism to reduce the "wall clock" time required to get a solution, principally by concurrent evaluations of the objective function. The implementations used here differ with respect to how many concurrent evaluations can be used effectively. In particular, two of the codes, PDS and GA, were designed explicitly to be executed in parallel and so they 
have the advantage that they can use any number of processors without any recoding. Given this implementation philosophy, the total number of function evaluations thes 2 methods take to reach a solution does not compare favorably with the sequential implementations of the other algorithms. However, when executed in parallel, the "wall clock" time for PDS and GA is more comjetitive. Nonetheless, for the tests reported here, MMF and some runs of DFO found feasible solutions with appreciably lower values of the objective and required far fewer total evaluations of the objective function in the process.

7. Conclusions. The results reported in Section 6 lend credence to our overall plan to develop approximation-based optimization methods that use SMF. Our current intent is to construct DACE approximations of sufficient accuracy that ANOVA decomposition will provide insight into the problem at hand. Subsequently, some variant of SMF, perhaps one with a BLGS flar or, will be used to identify the basin of a promising minimizer of the true objective function.

Of course, much remains to be done. We would like to find ways to accelerate the search for a minimizer after SMF has identified a basin and its reduction of the objective function has begun to slow. One possibility is then to use known objective function values to provide an initial approximation for DFO, as there are reasons to be believe that DFO enjoys faster local convergene: properties than SMF. Another important challenge is to extend SMF to address problems with general con itraints, particularly constraints that involve outputs of expensive analysis codes. We are currently working to address these issues.

Acknowledgments. The computational results presented in this paper were enriched significantly by the cooperation and collaboration of Katya Scheinberg in providing DFO test results. Our results showing that SMF can work with polynomial as well as DACE approxinations would have been much more difficult without the cooperation of Tom Grandine, who provided his implementation of the least-degree polynomial interpolant [12]. We also thank David Levine for suggesting parameter values for use in PGAPack.

Much of this work was accomplished during the year John Eennis and David Serafini spent in the Mathematics and Engineering Analysis Group at Boeing Shared Serv ices Group Applied Research \& Technology Division in Seattle. They wish to thank DOE and AFOSR resper tively for support, and the Boeing Company for the hospitality they were shown.

Virginia Torczon and Michael Trosset wish to thank AFGSR, NASA, and NSF for support and the Bocing Company for the hospitality that they were shown during their visits as part of this collaboration.

\section{REFERENCES}

[1] J.-F. M. BARThelemy ANd R. T. HAFtKa, Approximatic $n$ concepts for optimum structural design-a review, Structural Optimization, 5 (1993), pp. $129-144$

[2] A. J. BOOKER, DOE for computer output, Tech. Report BCSTECH 94 052, Bocing Computer Services, Research and Technology, M/S 7L-68, Seattle, Washin, ston 98124, December 1994.

[3] - Case studies in design and analysis of computer ex seriments, in Proceedings of the Section on Physical and Engineering Sciences, American Statisticel Association, 1996.

[4] A. J. Booker, A. R. Conn, J. E. Dennis, P. D. Frank, M. Trosset, And V. Torczon, Global modeling for optimization: Boeing/IBM/Rice collaborative project. 1995 final report, Tech. Report ISSTECH 95-032, Boeing Information \& Support Services, Research and Technology, M/S 7L-68, Seattle, Washington 98124, December 1995.

[5] A. J. Booker, J. E. Dennis, Jr., P. D. Frank, D. B. Serafini, and V. Torczon, Optimization 
using surrogate objectives on a helicopter test example, in Optimal Design and Control, J. Borggaard, J. Burns, E. Cliff, and S. Schreck, eds., Birkhauser, Cambridge, Massachusetts, to appear.

[6] S. Burgee, A. A. Giunta, V. Balabanov, B. Grossman, W. H. Mason, R. Narducci, R. T. HAFTKA, AND L. T. WATSON, A coarse grained parallel variable-complexity multidisciplinary optimization paradigm, The International Journal of Supcrcomputing Applications and High Performance Computing, 10 (1996), pp. 269299.

[7] A. R. Conn, K. SCheinberg, And P. L. Toint, On the convergence of derivative-free methods for unconstrained optimization, in Approximation Theory and Optimization: Tributes to M.J.D. Powell, M. D. Buhmann and A. Iserles, eds., Cambridge University Press, Cambridge, United Kingdom, 1997, pp. 83108.

[8] A. R. Conn and P. L. ToInt, An algorithm using quadratic interpolation for unconstrained derivative free optimization, in Nonlinear Optimization and Applications, G. Di Pillo and F. Giannessi, eds., Plenum Publishing, New York, 1996, pp. 27-47. Proceedings of the International School of Mathematics "G. Stampacchia" 21st Workshop, Erice, Italy, June 13 21, 1995.

[9] D. D. CoX AND S. JoHN, SDO: A statistical method for global optimization, in Multidisciplinary Design Optimization: State-of-the-Art, N. M. Alexandrov and M. Y. Hussaini, eds., SIAM, Philadelphia, 1997 , pp. 315329.

[10] C. Currin, T. Mitchell, M. Morris, And D. Ylvisaker, A Bayesian approach to the design and analysis of computer experiments, Tech. Report ORNL-6498, Oak Ridge National Laboratory, 1988.

[11] C. DAvis, Theory of positive linear dependence, American Journal of Mathematics, 76 (1954), pp. 733 746.

[12] C. DE Boor AND A. Ron, Computational aspects of polynomial interpolation in several variables, Mathematics of Computation, 58 (1992), pp. 705727.

[13] J. E. Dennis AND V. TorCzon, Managing approximation models in optimization, in Multidisciplinary Design Optimization: State-of-the-Art, N. M. Alexandrov and M. Y. Hussaini, eds., SIAM, Philadelphia, 1997, pp. 330-347.

[14] J. E. Dennis, JR. And V. Torczon, Direct search methods on parallel machines, SIAM Journal on Optimization, 1 (1991), pp. 448-474.

[15] J. E. Dennis, JR. AND H. F. WALKeR, Inaccuracy in quasi-Newton methods: Local improvement theorems, Mathematical Programming Study, 22 (1984), pp. 70-85.

[16] L. C. W. Dixon And G. P. Szegö, cds., Towards Global Optimisation, 2, North-Holland Publishing Company, Amsterdam, 1978.

[17] B. Efron and C. Stein, The jackknife estimate of variance, The Annals of Statistics, 9 (1981), pp. 586596.

[18] P. D. Frank, Global modeling for optimization, SIAG/OPT Views-and-News: A Forum for the SIAM Activity Group on Optimization, 7 (1995), pp. 9-12.

[19] S. GeIsser AND W. F. EdDy, A predictive approach to model selection, Journal of the American Statistical Association, 74 (1979), pp. 153-160.

[20] A. A. Giunta, Aircraft Multidisciplinary Design Optimization using Design of Experiments Theory and Response Surface Modeling Methods, PhD thesis, Department of Aerospace and Ocean Engineering, Virginia Polytechnic Institute \& State University, Blacksburg, Virginia, 1997; available as MAD 97 05 01, Multidisciplinary Analysis and Design Center for Advanced Vehicles, Virginia Polytechnic Institute \& State University, Blacksburg, Virginia 24061-0203. 
[21] D. R. Jones, M. Schonlau, And W. J. Welch, A data analytic approach to Bayesian global optimization, in Proceedings of the American Statistical Association, 1997.

[22] J. R. Koenler and A. B. Owen, Computer experimeits, in Design and analysis of experiments, S. Ghosh and C. R. Rao, cds., vol. 13 of Handbook of Statistics, North-Holland Publishing Company, Amsterdam, 1996, pp. 261308.

[23] D. LEVINE, Users guide to the PGAPack parallel genetic algorithm library, Tech. Report ANL-95/18, Argonne National Laboratory, 9700 South Cass Avenur:, Argonne, Illinois 60439, January 1996.

[24] R. M. LEwIS AND V. J. TORCZON, Pattern search algorithms for bound constrained minimization, Tech. Report 96 20, Institute for Computer Applications in Science and Enginecring, Mail Stop 403, NASA Langley Research Center, Hampton, Virginia 23681 2199, March 1996. To appear in SIAM Journal on Optimization.

[25] _ - Rank ordering and positive bases in pattern search clgorithms, Tech. Report 96-71, Institute for Computer Applications in Science and Engineering, Mail Stop 403, NASA Langley Research Center, Hampton, Virginia 23681 2199, 1996. In revision for Mathematical Programming.

[26] _ - Pattern search methods for linearly constrained mitimization, Tech. Report 98-3, Institute for Computer Applications in Science and Engineering, Mail Stop 403, NASA Langley Research Center, Hampton, Virginia 23681 2199, January 1998. To appear in SIAM Journal on Optimization.

[27] M. D. MCKay, W. J. Conover, and R. J. Beckman, A comparison of three methods for selecting values of input variables in the analysis of output from a computer code, Technometrics, 21 (1979), pp. $239-245$.

[28] A. B. OWEN, Orthogonal arrays for computer experimerts, integration and visualization, Statistica Sinica, 2 (1992), pp. 439452.

[29] A. H. G. Rinnooy Kan And G. T. TIMmer, A stochasti approach to global optimization, in Numerical Optimization 1984: Proceeding of the SIAM Conference on Numerical Optimization, Boulder, Colorado, June 12-14, 1984, SIAM, Philadelphia, 1985 pp. 245-262.

[30] B. D. RIPley, Statistical Inference for Spatial Processes, Cambridge University Press, Cambridge, United Kingdom, 1988.

[31] J. Sacks, W. J. Welch, T. J. Mitchell, And H. P. Wynn, Design and analysis of computer experiments, Statistical Science, 4 (1989), pp. 409423.

[32] D. B. SerafinI, A Framework for Managing Models in Optimization of Computationally Expensive Functions, PhD thesis, Department of Computational and Applied Mathematics, Rice University, P.O. Box 1892, Houston, Texas 77005 1892, 1998.

[33] L. A. Shultz, B. Panda, F. J. Tarzanin, R. C. Di:Rham, B. K. Oh, and L. Dadone, Interdisciplinary analysis for advanced rotors - approach, capabilities and status, in Proceedings of the American Helicopter Society Acromechanics Speci lists Conference, San Francisco, California, January 19 21, 1994, AHS, 1994, pp. PS 41 PS 415.

[34] M. Snir, S. W. Otto, S. Huss-Lederman, D. W. Wa: iker, and J. Dongarra, MPI: The Complete Reference, The MIT Press, Cambridge, Massachu: etts, 1996.

[35] M. StEIn, Large sample properties of simulations using Latin hypercube sampling, Technometrics, 29 (1987), pp. 143151.

[36] B. TANG, Orthogonal array-based Latin hypercubes, Journal American Statistical Association, 88 (1993), pp. $1392-1397$.

[37] V. TORCzon, PDS: Direct search methods for unconstrained optimization on either sequential or par- 
allel machines, Tech. Report TR92 09, Rice University, Department of Computational and Applied Mathematics, Houston, Texas 77005 1892, 1992. In revision.

[38] — - Pattern search methods for nonlinear optimization, SIAG/OPT Views-and-News: A Forum for the SIAM Activity Group on Optimization, 6 (1995), pp. 7-11.

[39] _ On the convergence of pattern search algorithms, SIAM Journal on Optimization, 7 (1997), pp. 1 25.

[40] V. Torczon AND M. W. Trosset, From evolutionary operation to parallel direct search: Pattern search algorithms for numerical optimization, Computing Science and Statistics, 29 (1998), pp. 396 401.

[41] M. W. Trosset, I know it when I see it: Toward a definition of direct search methods, SIAG/OPT Views-and-News: A Forum for the SIAM Activity Group on Optimization, 9 (1997), pp. 710.

[42] M. W. Trosset AND V. ToRCzon, Numerical optimization using computer experiments, Tech. Report 97 38, Institute for Computer Applications in Science and Enginecring, Mail Stop 403, NASA Langley Research Center, Hampton, Virginia 23681 2199, 1997.

[43] G. S. Watson, Smoothing and interpolation by kriging and with splines, Mathematical Geology, 16 (1984), pp. 601-615. 


\begin{tabular}{|c|c|c|c|}
\hline \multicolumn{3}{|c|}{ REPORT DOCUMENTATION PAGE } & $\begin{array}{l}\text { Form Approved } \\
\text { OMB No. 0704-0188 }\end{array}$ \\
\hline \multicolumn{4}{|c|}{ 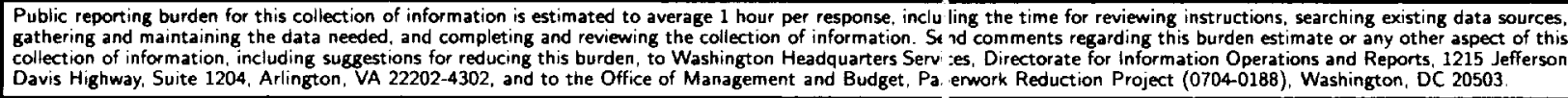 } \\
\hline 1. AGENCY USE ONLY(Leave blank) & $\begin{array}{l}\text { 2. REPORT DATE } \\
\text { November } 1998\end{array}$ & \multicolumn{2}{|c|}{$\begin{array}{l}\text { 3. REPOI:T TYPE AND DATES COVERED } \\
\text { Contr zctor Report }\end{array}$} \\
\hline \multicolumn{3}{|c|}{$\begin{array}{l}\text { 4. TITLE AND SUBTITLE } \\
\text { A Rigorous Framework for Optimization of Expensive Functions by } \\
\text { Surrogates }\end{array}$} & \multirow{2}{*}{$\begin{array}{l}\text { 5. FUNDING NUMBERS } \\
\text { C NAS1-19480 } \\
\text { C NAS1-97046 } \\
\text { WU 505-90-52-01 }\end{array}$} \\
\hline \multicolumn{3}{|c|}{$\begin{array}{l}\text { 6. AUTHOR(S) } \\
\text { Andrew J. Booker, J.E. Dennis, Jr., Paul D. Frank, } \\
\text { David B. Serafini, Virginia Torczon, and Michacl W. Trosset }\end{array}$} & \\
\hline \multicolumn{3}{|c|}{$\begin{array}{l}\text { 7. PERFORMING ORGANIZATION NAME(S) AND ADDRESS(ES) } \\
\text { Institute for Computer Applications in Science and Engincering } \\
\text { Mail Stop 403, NASA Langley Research Center } \\
\text { Hampton, VA } 23681-2199\end{array}$} & $\begin{array}{l}\text { 8. PERFORMING ORGANIZATION } \\
\text { REPORT NUMBER } \\
\text { ICASE Report No. } 98-47\end{array}$ \\
\hline \multicolumn{3}{|c|}{$\begin{array}{l}\text { 9. SPONSORING/MONITORING AGENCY NAME(S) AND ADDRESS(ES) } \\
\text { National Aeronautics and Space Administration } \\
\text { Langley Research Center } \\
\text { Hampton, VA } 23681-2199\end{array}$} & $\begin{array}{l}\text { 10. SPONSORING/MONITORING } \\
\text { AGENCY REPORT NUMBER } \\
\text { NASA/CR-1998-208735 } \\
\text { ICASE Report No. } 98-47\end{array}$ \\
\hline \multicolumn{4}{|c|}{$\begin{array}{l}\text { 11. SUPPLEMENTARY NOTES } \\
\text { Langley Technical Monitor: Dennis M. Bushnell } \\
\text { Final Report } \\
\text { To appear in Structural Optimization }\end{array}$} \\
\hline \multicolumn{2}{|c|}{$\begin{array}{l}\text { 12a. DISTRIBUTION/AVAILABILITY STATEMENT } \\
\text { Unclassified Unlimited } \\
\text { Subject Category } 64 \\
\text { Distribution: Nonstandard } \\
\text { Availability: NASA-CASI (301)621-0390 }\end{array}$} & 12b. DISTR & 12b. DISTRIBUTION CODE \\
\hline \multicolumn{4}{|c|}{$\begin{array}{l}\text { 13. ABSTRACT (Maximum } 200 \text { words) } \\
\text { The goal of the research reported here is to develop rigorous optimzation algorithms to apply to some engineering } \\
\text { design problems for which design application of traditional optimization approaches is not practical. This paper } \\
\text { presents and analyzes a framework for generating a sequence of approximations to the objective function and } \\
\text { managing the use of these approximations as surrogates for optimization. The result is to obtain convergence } \\
\text { to a minimizer of an expensive objective function subject to simple constraints. The approach is widely applicable } \\
\text { because it does not require, or even explicitly approximate, derivatives of the objective. Numerical results are } \\
\text { presented for a } 31 \text {-variable helicopter rotor blade design example ard for a standard optimization test example. }\end{array}$} \\
\hline \multirow{2}{*}{\multicolumn{3}{|c|}{$\begin{array}{l}\text { 14. SUBJECT TERMS } \\
\text { approximation concepts; surrogate optimization; response surfar es; pattern search } \\
\text { methods; derivative-free optimization; design and analysis of cor lputer experiments } \\
\text { (DACE); computational engineering }\end{array}$}} & $\begin{array}{l}\text { 15. NUMBER OF PAGES } \\
24\end{array}$ \\
\hline & & & \begin{tabular}{|c} 
16. PRICE CODE \\
$\mathrm{A} 03$
\end{tabular} \\
\hline $\begin{array}{l}\text { 17. SECURITY CLASSIFICATION } \\
\text { OF REPORT } \\
\text { Unclassified }\end{array}$ & $\begin{array}{l}\text { 18. SECURITY CLASSIFICATION } \\
\text { OF THIS PAGE } \\
\text { Unclassified }\end{array}$ & $\begin{array}{l}\text { 19. SECU RITY CLASSIFICATION } \\
\text { OF ABSTRACT }\end{array}$ & $\begin{array}{l}\text { 20. LIMITATION } \\
\text { OF ABSTRACT }\end{array}$ \\
\hline
\end{tabular}

